\title{
Continuous Galerkin Finite Element Methods for a Forward-Backward Heat Equation
}

\author{
Donald A. French * \\ Department of Mathematical Sciences, University of Cincinnati, PO Box 210025, Cincinnati, OH 45221
}

July 8, 1998

\begin{abstract}
A space-time finite element method is introduced to solve a model forward-backward heat equation. The scheme uses the continuous Galerkin method for the time discretization. An error analysis for the method is presented.
\end{abstract}

\section{Introduction:}

Consider the following forward-backward initial/boundary value problem: find $u=u(x, t)$ given $f=f(x, t)$ such that

$$
x u_{t}-u_{x x}=f \quad \text { in } \Omega=(-1,1) \times(0,1)
$$

with boundary conditions (BC)

$$
u=0 \text { on } \Sigma_{+} \cup \Sigma_{-}
$$

and initial/post conditions (IC/PC)

$$
u=0 \text { on } \Gamma_{+}^{0} \cup \Gamma_{-}^{1},
$$

where

$\Sigma_{+}=\{(1, t): t \in(0,1)\}, \quad \Sigma_{-}=\{(-1, t): t \in(0,1)\}, \quad \Gamma_{+}=\{x: x \in(0,1)\}, \quad \Gamma_{-}=\{x: x \in(-1,0)\}$,

and, in particular $\Gamma_{+}^{0}$ is at $t=0$, and $\Gamma_{+}^{1}$ is at $t=1$. We will often use the notation $\Gamma=(-1,1)$.

This equation arises in electron scattering (see [B]), fluid flow near boundary layers (see [GM]), and random acceleration of a particle (see $[\mathrm{FR}]$ ) (see also the discussion in [AFJK] for a full background). There have been several studies of the partial differential equation (see [BG] as

${ }^{*}$ Research supported in part by the Taft Foundation at the University of Cincinnati through their Grants-in-aid. 
well as the other papers listed in the references on this problem). Numerical work includes the investigation of a finite difference scheme in $[\mathrm{VK}]$ and methods using the transformation of (1) to a first order system were carried out in [AL1]. A least squares approach is described in [AL2]. In [AFJK] a two-dimensional finite element scheme for (1)-(3) is analyzed and in $[F]$ a discontinuous Galerkin(dG) finite element scheme is studied.

The continuous Galerkin(cG) time stepping scheme which we will study is closely related to the Gauss-Legendre Implicit Runge-Kutta schemes (IRK) (see [AM] and [FS] for more on cG methods and $[\mathrm{BDK}]$ for an application of IRK schemes to the KdV equation). The cG scheme can achieve the same high accuracy as the IRK scheme (see $[\mathrm{FP}]$ ) and because of its variational structure is amenable to the development of $a$ posteriori error estimates that are useful in adaptive computations (see $[\mathrm{EF}])$.

Closely related to the cG method is the dG method (see [T]). In an ordinary differential equation setting the cG method can achieve convergence rates proportional to $k^{2 m}$ at the nodes if there are $m$ degrees of freedom on each step with length $k$ while the dG method will achieve slightly lower rates that are proportional to $k^{2 m-1}$. This is nicely exemplified in the $m=1$ cases. The cG method is closely related to the Crank-Nicholson method $\left(O\left(k^{2}\right)\right)$ while the dG method is related to the backward Euler scheme $(O(k))$.

Our main result is on a uniform grid where the refinements by parameter $h>0$ in space and time are identical and the approximation spaces consist of piecewise polynomials of degree $\leq p$. We show that

$$
\left(\iint_{\Omega} x^{2} e(x, t)^{2} d x d t\right)^{1 / 2} \leq C h^{p+1}
$$

where $e$ is the error. Our general theorem will also include the case where space and time refinements could be different as could the degrees of the piecewise polynomial functions.

A weak formulation which is used to motivate the finite element scheme can be found by multiplying (1) by $v \in H_{0}^{1}(\Gamma)$ and integrating over $\Gamma$. The set $H^{m}(A)$ for some domain $A$ is the Sobolev space with functions that have $m$ derivatives in $L^{2}(A)$. The space $H_{0}^{1}(A)$ is functions in $H^{1}(A)$ which have trace zero on the $\partial A$. Integration-by-parts and the $\mathrm{BC}$ are used to rewrite the term involving $u_{x x}$,

$$
\left(x u_{t}, v\right)_{\Gamma}+\left(u_{x}, v_{x}\right)_{\Gamma}=(f, v)_{\Gamma}, \quad \forall v \in H_{0}^{1}(\Gamma),
$$

where the desired solution $u \in H_{0}^{1}(\Gamma)$ and for a domain $A$ we define the $L^{2}$ inner product

$$
(w, v)_{A}=\int_{A} w v d A \text { and norm }\|w\|_{A}^{2}=(w, w)_{A} .
$$

By setting $v=u$ we have

$$
\frac{1}{2} \frac{d}{d t}(x u, u)_{\Gamma}+\left\|u_{x}\right\|_{\Gamma}^{2}=(f, u)_{\Gamma} .
$$


Defining

$$
\|w\|_{-1, \Gamma}=\max _{v \in H_{0}^{1}(\Gamma)} \frac{(w, v)_{\Gamma}}{\left\|v_{x}\right\|_{\Gamma}}
$$

we obtain, using the inequality

$$
a b \leq \frac{\epsilon}{2} a^{2}+\frac{1}{2 \epsilon} b^{2}
$$

with $\epsilon=1$, that

$$
\frac{d}{d t}\left(x u^{2}, 1\right)_{\Gamma}+\left\|u_{x}\right\|_{\Gamma}^{2} \leq\|f\|_{-1, \Gamma}^{2}
$$

Integration with respect to $t$ over $[0,1]$ gives

$$
\left\|x^{1 / 2} u\right\|_{\Gamma_{+}^{1}}^{2}+\left\||x|^{1 / 2} u\right\|_{\Gamma_{-}^{0}}^{2}+\left\|u_{x}\right\|_{\Omega}^{2} \leq \int_{0}^{1}\|f\|_{-1, \Gamma}^{2} d t .
$$

This is a fundamental a priori estimate for the problem and one we will exploit in our analysis.

The estimate (7) shows that if (1)-(3) has a solution it will be unique. We will assume throughout that there is a unique solution which is as regular as required by an analysis. We will use the Poincaré inequality;

$$
\|u\|_{\Gamma} \leq C\left\|u_{x}\right\|_{\Gamma} \quad \text { for } \quad u \in H_{0}^{1}(\Gamma)
$$

in the succeeding sections.

\section{Approximation Method:}

In this section we introduce the space-time finite element scheme that uses the cG method for the time discretization.

We first specify the grids. Let $h=1 /(M+1)$ and $x_{j}=j h$ where $j=0, \pm 1, \pm 2, \ldots, \pm(M+1)$. Let $k=1 / N$ and $t_{j}=j k$ for $j=0,1, \ldots, N$.

We now turn to the definition of the approximation spaces. We will keep careful track of dimension to ensure that the resulting problem leads to a square system of equations. Let $X_{h}^{p}$ be the space of continuous piecewise polynomials of degree $\leq p$ on the spatial grid which are zero at \pm 1 . Subtracting the continuity and boundary condition constraints from the degrees of freedom we find that $\operatorname{dim} X_{h}^{p}=(2 M+2) p-1$. For the temporal discretization we let $C_{k}^{q}$ be the set of continuous piecewise polynomials of degree $\leq q$ and $D_{k}^{r}$ be piecewise polynomials, not necessarily continuous, of degree $\leq r$. Counting the constraints of $C_{k}^{q}$ and degrees of freedom for each we find that $\operatorname{dim} C_{k}^{q}=N q+1$ and $\operatorname{dim} D_{k}^{r}=N(r+1)$.

The solution space $S_{h k}$ will consist of all functions in $X_{h}^{p} \otimes C_{k}^{q}$ which are zero at all nodes on $\bar{\Gamma}_{-}^{1}$ and all nodes on $\bar{\Gamma}_{+}^{0}$ except $(0,0)$. Thus

$$
\begin{aligned}
\operatorname{dim} S_{h k} & =\left(\operatorname{dim} X_{h}^{p}\right) \times\left(\operatorname{dim} C_{k}^{q}\right)-\{\text { IC and PC constraints }\} \\
& =[(2 M+2) p-1][N q+1]-[(2 M+2) p-1] \\
& =[(2 M+2) p-1] N q .
\end{aligned}
$$


The test space is $T_{h k}=X_{h}^{p} \otimes D_{k}^{q-1}$ and

$$
\operatorname{dim} T_{h k}=\left(\operatorname{dim} X_{h}^{p}\right) \times\left(\operatorname{dim} D_{k}^{q-1}\right)=[(2 M+2) p-1] N q=\operatorname{dim} S_{h k} .
$$

With these notations the cG method is defined by the following variational problem: find $U \in S_{h k}$ such that

$$
\left(x U_{t}, \phi\right)_{\Omega}+\left(U_{x}, \phi_{x}\right)_{\Omega}=(f, \phi)_{\Omega} \quad \forall \phi \in T_{h k} .
$$

We will use several projection operators throughout. Define $\pi_{t}: L^{2}(\Sigma) \rightarrow D_{k}^{q-1}$ by

$$
\left(\pi_{t} v, \chi\right)_{\Sigma}=(v, \chi)_{\Sigma} \quad \forall \chi \in D_{k}^{q-1}
$$

where $\Sigma=(0,1)$. Define $P_{t}: H^{1}(\Sigma) \rightarrow C_{k}^{q}$ by

$$
\left(\left(P_{t} v\right)_{t}, \eta_{t}\right)_{\Sigma}=\left(v_{t}, \eta_{t}\right)_{\Sigma} \quad \forall \eta \in C_{k}^{q}
$$

with $P_{t} v(0)=v(0)$ and it then follows that $P_{t} v\left(t_{j}\right)=v\left(t_{j}\right)$ for $j=1,2, \ldots, N$. Finally, define $P_{x}: H_{0}^{1}(\Gamma) \rightarrow X_{h}^{p}$ by

$$
\left(\left(P_{x} v\right)_{x}, \lambda_{x}\right)_{\Gamma}=\left(v_{x}, \lambda_{x}\right)_{\Gamma} \quad \forall \lambda \in X_{h}^{p}
$$

and $\pi_{x}: L^{2}(\Gamma) \rightarrow X_{h}^{p}$ by

$$
\left(\left(\pi_{x} v\right), \psi\right)_{\Gamma}=(v, \psi)_{\Gamma} \quad \forall \psi \in X_{h}^{p} .
$$

Note, in particular, that $\pi_{t}$ and $P_{t}$ act independently of the spatial operators, inner products and functions while $P_{x}$ and $\pi_{x}$ act independently of the temporal operators, inner products, and functions.

We now proceed to give the approximation properties for the spaces and operators which we will use. See Ciarlet $[\mathrm{C}]$ for discussion and justification of these standard results. We will use the following inverse inequalities: for $\lambda \in P_{r}\left(I_{n}\right)$

$$
\|\lambda\|_{L^{\infty}\left(I_{n}\right)} \leq C k^{-1 / 2}\|\lambda\|_{I_{n}} \text { and }\left\|\lambda_{t}\right\|_{I_{n}} \leq C k^{-1}\|\lambda\|_{I_{n}}
$$

where $I_{n}=\left(t_{n-1}, t_{n}\right)$. For $\chi \in X_{h}^{p}$ we have

$$
\left\|\chi_{x}\right\|_{\Gamma} \leq C h^{-1}\|\chi\|_{\Gamma} \quad \text { and } \quad\left\|\left(\frac{d}{d x}\right)^{\ell} \chi\right\|_{L^{\infty}(\Gamma)} \leq C h^{-1 / 2}\left\|\left(\frac{d}{d x}\right)^{\ell} \chi\right\|_{\Gamma}, \quad \ell=0,1 .
$$

We now summarize the key properties we will use for the operators $\pi_{x}, \pi_{t}, P_{x}$, and $P_{t}$. For $v \in H^{s}(\Sigma)$ there is a constant $C$ such that

$$
\left\|\left(I-P_{t}\right) v\right\|_{\Sigma} \leq C k^{s}\|v\|_{s, \Sigma} \quad \text { and } \quad\left\|\left(I-\pi_{t}\right) v\right\|_{\Sigma} \leq C k^{r}\|v\|_{r, \Sigma}, \quad 0 \leq r, s \leq q+1 .
$$

The measure, $\|\cdot\|_{\ell, \Sigma}$ is the $H^{\ell}(\Sigma)$ norm. For the spatial operators we have

$$
\left\|\left(I-P_{x}\right) w\right\|_{\ell, \Gamma} \leq C h^{s-\ell}\|w\|_{s, \Gamma} \quad \text { and } \quad\left\|\left(I-\pi_{x}\right) w\right\|_{\ell, \Gamma} \leq C h^{r-\ell}\|w\|_{r, \Gamma}, \quad 0 \leq r \leq p+1, \ell=0,1 .
$$


We note that, in particular, $P_{x}$ is bounded in $L^{2}(\Gamma)$. It can also be shown using $P_{x}$, the inverse estimate (11), and the approximation properties (13) that

$$
\left\|\pi_{x} w\right\|_{1, S_{n}} \leq C\|w\|_{1, S_{n}} \quad \forall w \in H_{0}^{1},(\Gamma)
$$

where $S_{n}=\Gamma \times I_{n}$. The following lemma gives a useful bound involving $\pi_{t}$. Denote evaluation at time level $t_{n}$ with a superscript $n$.

Lemma 1: For any real number $\beta \geq 0$ there exists a constant $C$ such that

$$
\left\||x|^{\beta} V\right\|_{\Omega}^{2} \leq k \sum_{n=0}^{N}\left\||x|^{\beta} V^{n}\right\|_{\Gamma}^{2}+C\left\||x|^{\beta} \pi_{t} V\right\|_{\Omega}^{2} \quad \forall V \in X_{h}^{p} \otimes C_{k}^{q} .
$$

Proof: Adding the weight function $|x|^{\beta}$ to the proof in French and Jensen ([FJ] lemma 1, p. 427) we have

$$
\left\||x|^{\beta} V\right\|_{S_{n}}^{2} \leq k\left\||x|^{\beta} V^{n-1}\right\|_{\Gamma}^{2}+C\left\||x|^{\beta} \pi_{t} V\right\|_{S_{n}}^{2}
$$

and summing from 1 to $N$ we have

$$
\left\||x|^{\beta} V\right\|_{\Omega}^{2} \leq k \sum_{n=1}^{N}\left\||x|^{\beta} V^{n-1}\right\|_{\Gamma}^{2}+C\left\||x|^{\beta} \pi_{t} V\right\|_{\Omega}^{2}
$$

and from this (15) follows.

Finally, we note that the following trivial weight function inequality holds for any real number $\beta \geq 0$ :

$$
\left\||x|^{\beta} v\right\|_{\Gamma} \leq\|v\|_{\Gamma}
$$

\section{Preliminary Estimates:}

In this section we derive several estimates from the cG variational equation:

$$
\left(x V_{t}, \phi\right)_{S_{n}}+\left(V_{x}, \phi_{x}\right)_{S_{n}},=(g, \phi)_{S_{n}}
$$

where $V \in S_{h k}, \phi \in T_{h k}$, and $g \in L^{2}(\Omega)$ We will use these results to conclude (9) has a unique solution and, in the next section, to form an error estimate for this method.

Lemma 2: Suppose $V \in S_{h k}$ satisfies (17) then

$$
\left\|\pi_{t} V_{x}\right\|_{\Omega}^{2}+\left\||x|^{1 / 2} V\right\|_{\Gamma_{+}^{1}}^{2}+\left\||x|^{1 / 2} V\right\|_{\Gamma_{-}^{0}}^{2} \leq C\|g\|_{\Omega}^{2}
$$

Proof: Let $\phi=\pi_{t} V \in S_{h k}$ in (17), sum from $n=1, \ldots, N$, and observe that $V_{t} \in T_{h k}$ to see that

$$
\left(x V_{t}, V\right)_{\Omega}+\left(\pi_{t} V_{x}, \pi_{t} V_{x}\right)_{\Omega}=\left(g, \pi_{t} V\right)_{\Omega}
$$


From the Poincaré inequality (8), arithmetic-geometric mean inequality (6), and the IC/PC on $V$ we have

$$
\frac{1}{2}\left[\left(x V^{2}, 1\right)_{\Gamma_{+}^{1}}-\left(x V^{2}, 1\right)_{\Gamma_{-}^{0}}\right]+\left\|\pi_{t} V_{x}\right\|_{\Omega}^{2} \leq \frac{1}{4 \epsilon}\|g\|_{\Omega}^{2}+\epsilon\left\|\pi_{t} V\right\|_{\Omega}^{2} \leq \frac{1}{4 \epsilon}\|g\|_{\Omega}^{2}+C \epsilon\left\|\pi_{t} V_{x}\right\|_{\Omega}^{2} .
$$

The inequality (18) now follows from this by choosing $\epsilon=1 / C$. $\square$ The next lemma gives a bound on the approximation functions at the time nodes, $t_{n}$.

Lemma 3: Suppose $V \in S_{h k}$ satisfies (17) then there is a constant $C$ such that

$$
\left\|x V^{n}\right\|_{\Gamma}^{2} \leq C\left(\|x V\|_{\Gamma_{-}^{0}}^{2}+\|x V\|_{\Gamma_{+}^{1}}^{2}+\|g\|_{\Omega}^{2}+\left(1+\frac{h^{2}}{\epsilon k^{2}}\right)\left\|\pi_{t} V_{x}\right\|_{\Omega}^{2}\right)+\epsilon\|x V\|_{\Omega}^{2}
$$

where $0<\epsilon<1$.

Proof: Take $\phi=\pi_{x} \pi_{t}(x V)$ in (17) and obtain

$$
\left(x V_{t}, \pi_{x} \pi_{t}(x V)\right)_{S_{n}}=-\left(V_{x},\left(\pi_{x} \pi_{t}(x V)\right)_{x}\right)_{S_{n}}+\left(g, \pi_{x} \pi_{t}(x V)\right)_{S_{n}}
$$

or

$$
\left(x^{2} V_{t}, V\right)_{S_{n}}=-\left(\pi_{t} V_{x},\left(\pi_{x}\left(x \pi_{t} V\right)\right)_{x}\right)_{S_{n}}+\left(g, \pi_{x}\left(x \pi_{t} V\right)\right)_{S_{n}}+\left(x V_{t},\left(I-\pi_{x}\right)\left(x \pi_{t} V\right)\right)_{S_{n}} .
$$

Using the inverse inequality (10) and the arithmetic geometric mean inequality (6) we obtain

$$
\begin{aligned}
\frac{1}{2}\left\|x V^{n}\right\|_{\Gamma}^{2} \leq & \frac{1}{2}\left\|x V^{n-1}\right\|_{\Gamma}^{2}+\frac{1}{2}\|g\|_{S_{n}}^{2}+\frac{1}{2}\left\|\pi_{x}\left(x \pi_{t} V\right)\right\|_{S_{n}}^{2}+\left\|\pi_{t} V_{x}\right\|_{S_{n}}\left\|\pi_{x}\left(x \pi_{t} V\right)_{x}\right\|_{S_{n}} \\
& +C k^{-1}\|x V\|_{S_{n}}\left\|\left(I-\pi_{x}\right)\left(x \pi_{t} V\right)\right\|_{S_{n}} .
\end{aligned}
$$

Applying (6), the estimate (13), the Poincaré inequality (8), (14), and (16) we have

$$
\frac{1}{2}\left\|x V^{n}\right\|_{\Gamma}^{2} \leq \frac{1}{2}\left\|x V^{n-1}\right\|_{\Gamma}^{2}+\frac{1}{2}\|g\|_{S_{n}}^{2}+C\left(1+\frac{h^{2}}{\epsilon k^{2}}\right)\left\|\pi_{t} V_{x}\right\|_{S_{n}}^{2}+\epsilon / 2\|x V\|_{S_{n}}^{2} .
$$

Iterating this inequality we obtain

$$
\left\|x V^{n}\right\|_{\Gamma}^{2} \leq\left\|x V^{0}\right\|_{\Gamma}^{2}+\|g\|_{\Omega}^{2}+C\left(1+\frac{h^{2}}{\epsilon k^{2}}\right)\left\|\pi_{t} V_{x}\right\|_{\Omega}^{2}+\epsilon\|x V\|_{\Omega}^{2}
$$

The estimate (19) now follows by adding $\left\|x V^{n}\right\|_{\Gamma_{1}^{+}}$and noting that $V^{0} \equiv 0$ on $\Gamma_{0}^{+}$.

We now are in a position to derive a crucial estimate on the approximation function $V$.

Theorem 1: Suppose $V \in S_{h k}$ satisfies (17) then there is a constant $C$ such that

$$
\|x V\|_{\Omega} \leq C\left(1+\frac{h^{2}}{k^{2}}\right)\|g\|_{\Omega}
$$


Proof: From lemma 1 with $\beta=1$, the weight function inequality (16), and the Poincaré inequality (8) we have

$$
\|x V\|_{\Omega}^{2} \leq k \sum_{n=0}^{N}\left\|x V^{n}\right\|_{\Gamma}^{2}+C\left\|\pi_{t} V_{x}\right\|_{\Omega}^{2}
$$

Using (19) we obtain

$$
\|x V\|_{\Omega}^{2} \leq k N\left[C\left(\|x V\|_{\Gamma_{-}^{0}}^{2}+\|x V\|_{\Gamma_{+}^{1}}^{2}+\|g\|_{\Omega}^{2}+\left(1+\frac{C h^{2}}{\delta k^{2}}\right)\left\|\pi_{t} V_{x}\right\|_{\Omega}^{2}\right)+\delta\|x V\|_{\Omega}^{2}\right]+C\left\|\pi_{t} V_{x}\right\|_{\Omega}^{2},
$$

where $\delta=C \epsilon$. Noting that $k N=1$, choosing $\delta=1 / 2$, observing that $|x| \leq|x|^{1 / 2}$ on $[-1,1]$, and once again using (16) as well as the result of lemma 2 we obtain (20).

This estimate allows us to conclude the approximation problem (9) has a unique solution. As noted before (9) leads to a square system of linear equations. By considering the possibility of two solutions we are lead to evaluate the problem with $f=0$. From (20) we see that the only solution of the homogeneous problem is the zero solution. Thus, we conclude (9) has a unique solution.

\section{Main Results:}

In this section we present the main result of this paper. We will use a decomposition of the error involving projection operators similar to standard ones from [T]. The final estimate will involve the norm

$$
N(u)^{2}=\sum_{\ell=0}^{1}\left\|\left(\frac{d}{d t}\right)^{\ell} u\right\|_{L^{2}\left(I ; H^{p+1}(\Gamma)\right)}^{2}+\sum_{\ell=0}^{2}\left\|\left(\frac{d}{d x}\right)^{\ell} u\right\|_{H^{q+1}\left(I ; L^{2}(\Gamma)\right)}^{2}
$$

and the rate quantity

$$
\gamma(h, k)=\left(h^{2 p+2}+k^{2 q+2}\right)^{1 / 2} N(u) .
$$

Theorem 2: Suppose $u$ is the solution of (1)-(3) and is sufficiently smooth so $N(u)<\infty$. Also suppose $U$ is the solution of (9) then

$$
\|x(u-U)\|_{\Omega} \leq C\left(1+\frac{h^{2}}{k^{2}}\right) \gamma(h, k)
$$

Proof: Let

$$
u-U=\left(u-P_{x} P_{t} u\right)+\left(P_{x} P_{t} u-U\right)=\rho+\theta .
$$

From the inequality (16) and the approximation properties of the $P_{x}$ and $P_{t}$ operators it follows that

$$
\|x \rho\|_{\Omega} \leq\left\|u-P_{x} u\right\|_{\Omega}+\left\|P_{x}\left(I-P_{t}\right) u\right\|_{\Omega} \leq C \gamma(h, k) .
$$


Note that $\theta \in S_{h k}$. For $\phi \in T_{h k}$ we have

$$
\begin{aligned}
\left(x \theta_{t}, \phi\right)_{S_{n}}+\left(\theta_{x}, \phi_{x}\right)_{S_{n}} & =\left(x\left(P_{t} P_{x} u\right)_{t}, \phi\right)_{S_{n}}+\left(\left(P_{t} P_{x} u\right)_{x}, \phi_{x}\right)_{S_{n}}-(f, \phi)_{S_{n}} \\
& =\left(x P_{x} u_{t}, \phi\right)_{S_{n}}+\left(P_{t} u_{x}, \phi_{x}\right)_{S_{n}}-\left(x u_{t}, \phi\right)_{S_{n}}-\left(u_{x}, \phi_{x}\right)_{S_{n}} \\
& \left.=\left(x\left(P_{x}-I\right) u_{t}, \phi\right)_{S_{n}}+\left(\left(P_{t}-I\right) u\right)_{x}, \phi_{x}\right)_{S_{n}} \\
& =(R, \phi)_{S_{n}}
\end{aligned}
$$

where

$$
R=x\left(P_{x}-I\right) u_{t}-\left(P_{t}-I\right) u_{x x} .
$$

From (20) and the approximation properties of $P_{x}$ and $P_{t}$ we have

$$
\|x \theta\|_{\Omega} \leq C\left(1+\frac{h^{2}}{k^{2}}\right)\|R\|_{\Omega} \leq C\left(1+\frac{h^{2}}{k^{2}}\right) \gamma(h, k) .
$$

Combining this with (22) proves the theorem.

\section{Numerical Results:}

In this section we describe the results of two computational experiments with the cG finite element scheme set in the simplest case when $p=q=1$ and $h=k$. The examples are designed so that the true solution is known. This allows us to verify that the weighted error, $\|x(u-U)\|_{\Omega}$, will tend to zero at rate $O\left(h^{2}\right)$.

We first briefly describe our implementation. Since the test functions in the $q=1$ case are constant in $t$ on each slab, $S_{n}$, it can be shown that (9) in the $p=q=1$ is equivalent to

$$
k^{-1}\left(x\left(U^{n+1}-U^{n}\right), \chi\right)_{\Gamma}+\frac{1}{2}\left(\left(U^{n}+U^{n+1}\right)_{x}, \chi_{x}\right)_{\Gamma}=\left(k^{-1} \int_{t_{n}}^{t_{n+1}} f(\cdot, t) d t, \chi\right)_{\Gamma}
$$

for $n=0,1, \ldots, N-1$ and $\chi \in X_{h}^{1}$. We used Simpson's rule to compute the inner product and time integrations that occur on the right side of the equation. Since this rule is very accurate at $O\left(h^{4}\right)$ we do not expect the approximation of this term will alter the convergence rate of the overall scheme.

There are many possible approaches to solving the equations that arise in numerical approximation schemes for forward-backward parabolic problems (see [VK] for an iterative scheme that solves the forward and backward parts separately and [AFJK] for a full list of possible schemes). Since our focus is on verifying the convergence theorem we take the test functions to be the elements in the usual hat function basis for $X_{h}^{1}$ and obtain a system equations for the unknown values of $U$ at the nodes, $U_{j}^{n}=U\left(x_{j}, t_{n}\right)$. We solve the resulting system using Gaussian Elimination.

In the succeeding two experiments we chose a function for $u$, substituted it into (1), and obtained a right side function $f$. The integrations in the computations of the $L^{2}(\Omega)$-norm of the errors are 
approximated using the Trapezoid rule in each example. The solutions in each case are smooth guarranteeing that $N(u)<\infty$. In example 1 the function $u$ satisfies the $\mathrm{BC} / \mathrm{IC} / \mathrm{PC}$ and thus the theorem applies with the exception of the Simpson's rule approximation on the right side term. In example 2 the theorem does not apply since the known solution does not satisfy the BC/IC/PC. However in both cases we see that the ratio of the error to $h^{2}$ is tending to a constant confirming the theorem.

Example 1: $u(x, y)=\cos (\pi x / 2) \sin (\pi x)$.

\begin{tabular}{|c|c|c|}
\hline$h$ & $\|x(u-U)\|_{\Omega}$ & $\|x(u-U)\|_{\Omega} / h^{2}$ \\
\hline $1 / 2$ & $5.9074(-2)$ & 0.236 \\
$1 / 4$ & $1.3324(-2)$ & 0.213 \\
$1 / 8$ & $3.2163(-3)$ & 0.206 \\
$1 / 16$ & $7.9725(-4)$ & 0.204 \\
\hline
\end{tabular}

Example 2: $u(x, y)=\cos (x) \cos (x)$.

\begin{tabular}{|c|c|c|}
\hline$h$ & $\|x(u-U)\|_{\Omega}$ & $\|x(u-U)\|_{\Omega} / h^{2}$ \\
\hline $1 / 2$ & $3.6568(-3)$ & 0.0146 \\
$1 / 4$ & $7.7141(-4)$ & 0.0123 \\
$1 / 8$ & $1.8442(-4)$ & 0.0118 \\
$1 / 16$ & $4.5577(-5)$ & 0.0117 \\
\hline
\end{tabular}

\section{Conclusions:}

In this paper we have described a space-time finite element method for a forward-backward parabolic problem and proved an optimal a priori error estimate in a weighted norm. We have also performed some computations that display this result.

We now compare our convergence result to others in the literature. The equation we study has the form $\sigma u_{t}-u_{x x}=f$. We have restricted our analysis to the case where $\sigma(x, t)=x$ in order to keep the arguments straightforward. Here, we have shown $O\left(h^{p+1}\right)$ convergence in a weighted $L^{2}$-norm. In [VK] a first-order in time, second-order in space, finite difference method is analyzed and the $L^{\infty}$-norm of the error at the nodes is shown to converge at rate $O\left(h^{2}+k\right)$ under the restriction that $\sigma=\sigma(x)$. In [AL1], [AL2], and [AFJK] the function $\sigma$ is allowed to depend on $x$ and $t$. A rate of $O\left(h^{p}\right)$ was shown for the $L^{2}(\Omega)$-norm of the error in [AL1] and [AL2]. This $O\left(h^{p}\right)$ rate was shown for the $L^{2}(\Omega)$-norm of the $x$-derivatives of the error in [AL2] and [AFJK] which is optimal. We showed $O\left(h^{p}\right)$ convergence for the $L^{2}$-norm of $x$-derivative of the error in [F] where the $\mathrm{dG}$ finite element method is considered with $\sigma(x, t)=x$. 
We anticipate that some generalizations of our theorem are possible. We expect that if $\sigma$ is independent of $t$ and smooth the argments above can be generalized with different weight functions. However we expect the dependence of $\sigma$ on $t$ must be restricted so the curves separating regions where $\sigma>0$ from those where $\sigma<0$ are parallel or close to parallel the $t$-axis. In contrast, we expect that the $\mathrm{dG}$ scheme (see $[\mathrm{F}]$ ) will generalize mainly because one does not have to count nodes as carefully as was needed for cG (see section 2).

\section{References}

[AFJK] A.K. Aziz, D.A. French, S. Jensen, and R.B. Kellogg, Origins, analysis, and numerical analysis of a forward-backward parabolic problem (submitted to $M^{2} A N$ ).

[AL1] A.K. Aziz and J.-L. Liu, A Galerkin method for the forward-backward heat equation, Math. Comp. 56 (1991), 35-44.

[AL2] A.K. Aziz and J.-L. Liu, A weighted least squares method for the backward-forward heat equation, SIAM J. Num. Anal. 28 (1991), 156-167.

[AM] A.K. Aziz and P. Monk, Continuous finite elements in space and time for the heat equation, Math. Comp. 52 (1989), 255-274.

[BDK] J.L. Bona, V.A. Dougalis, and O.A. Karakashian, Fully discrete Galerkin methods for the Kortewig-de-Vries equation, Comput. Math. Appl. 12A (1986), 859-884.

[BG] M.S. Baouendi and P. Grisvard, Sur une équation d'evolution changeant de type, J. Funct. Anal. 28 (1968), 352-367.

[B] R. Beals, An abstract treatment of some forward backward problems in transport and scattering, J. Funct. Anal. 34 (1979), 1-20.

[C] P.G. Ciarlet, The finite element method for elliptic problems, North Holland, Amsterdam (1978).

[EF] D. Estep and D.A. French, Global error control for the continuous Galerkin finite element method for ordinary differential equations, RAIRO 28 (1994), 815-852.

[FR] J.N. Franklin and E.R. Rodemich, Numerical analysis of an elliptic-parabolic partial differential equation, SIAM J. Num. Anal 5 (1968), 680-716.

[F] D.A. French, Discontinuous Galerkin finite element methods for a forward-backward heat equation, Appl. Numer. Math. 27 (1998), 1-8. 
[FJ] D.A. French and S. Jensen, Long-time behaviour of arbitrary order continuous time Galerkin schemes for some one-dimensional phase transition problems, IMA J. Num. Anal. 14 (1994), 421-442.

[FP] D.A. French and T.E. Peterson, A continuous space-time finite element method for the wave equation, Math. Comp., 65 (1996), 491-506.

[FS] D.A. French and J.W. Schaeffer, Continuous finite element methods which preserve energy properties for nonlinear problems, Appl. Math. Comp. 39 (1990), 271-295.

[GM] J.A. Goldstein and T. Mazumdar, A heat equation in which the diffusion coefficient changes sign, J. Math. Anal. Appl. 103 (1984), 533-564.

[T] V. Thomée, Galerkin Finite Element Methods for Parabolic Problems, Lecture Notes in Mathematics \# 1054, Springer Verlag, Berlin (1984).

[VK] V. Vanaja and R.B. Kellogg, Iterative methods for a forward-backward heat equation, SIAM J. Num. Anal. 27 (1990), 622-635. 